\title{
Exponential stabilization for nonlinear switched stochastic systems with interval time-varying delay under asynchronous switching
}

\section{Yongzhao Wang ${ }^{1 *}$ (1)}

\author{
"Correspondence: \\ wangyongzhao1987@126.com \\ 'School of Mathematics and \\ Statistics, Anyang Normal University, \\ Anyang, China
}

\begin{abstract}
The paper investigates mean-square exponential stabilization for a class of nonlinear switched stochastic systems with interval time-varying delay under asynchronous switching. Specifically, the delay occurs not only in the state equation, but also in the switching signal from the controller, which brings the difficulty of controller design to achieve mean-square exponential stabilization. Based on the Lyapunov stability theory, a new piecewise multi-Lyapunov-Krasovskii functional dependent on the size of time delay is constructed. By utilizing the matrix inequality technique and the average dwell time approach, delay-dependent sufficient conditions are given to guarantee mean-square exponential stabilization for nonlinear switched stochastic systems under asynchronous switching. In accordance with the method, we also design state feedback controllers of the switched stochastic systems under asynchronous switching through special operations of matrices and Schur complement. Finally, a numerical example and a practical example of river pollution control are provided to show the effectiveness of the approach proposed in this paper.
\end{abstract}

MSC: 93D20; 93E10; 93C10; 34D20

Keywords: Switched stochastic system; Asynchronous switching; Interval time-varying delay; Average dwell time; Lyapunov Krasovskii functional

\section{Introduction}

During the last two decades, hybrid systems have become increasingly important in contemporary society both in science and technology due mainly to the fact that hybrid systems have been extensively applied in many fields such as pattern recognition [1], network control [2], power systems [3], automotive systems [4], communication systems [5], neural networks [6], and so on. A switched system is one of the special dynamic hybrid systems that comprise a collection of subsystems equipped with a switching law orchestrating among these systems. Many of switched system models appear in the fields of industrial manufacturing, artificial intelligence, biochemical systems, actuator failures [7], and population dynamics [8-10].

(c) The Author(s) 2019. This article is distributed under the terms of the Creative Commons Attribution 4.0 International License (http://creativecommons.org/licenses/by/4.0/), which permits unrestricted use, distribution, and reproduction in any medium, provided you give appropriate credit to the original author(s) and the source, provide a link to the Creative Commons license, and indicate if changes were made. 
The analysis and synthesis are important issues in the study of switched systems, and they have attracted extensive attention from domestic and foreign scientific research. So far, much progress has been made and many remarkable achievements for various types of switched systems have been studied. For example, behavior analysis [11, 12], property characterization [13], fault detection [14], and control synthesis [15-17]. These results show that stability is a crucial and fundamental problem for switched system with time delay. Essentially, time delay, perturbation, and stochastic term are common phenomena often encountered in real dynamical systems [18-20]. Moreover, the effect of time delays generally exists in system states and will be a source of control system instability, oscillation, and performance deterioration. As a result, the study of delay and stochastic term plays an important role in the stability analysis of switched system. With respect to those problems, we just mention here some representative work. In [21], a new method of uncertain matrix was proposed. Based on this approach, an exponential stabilization condition of nonlinear uncertain systems with time-varying delay was firstly established. By following this idea, [22] also studied the delay-dependent stability analysis and relevant control problems for nonlinear switched with interval time-varying delay based on Lyapunov-Krasovskii functional method. Robust guaranteed cost control for a class of uncertain neutral system with time-varying delays was investigated in [23], delay-dependent and delay-independent criteria were proposed for the stabilization of considered systems, state feedback control was considered to stabilize the uncertain neutral system, and upper bounds on the closed-loop cost function were also given. [24] obtained sufficient conditions with delay-dependent guaranteeing the exponential stability by a common Lyapunov functional (CLF). Recently analogous results have been found in [25], and [25] constructed a suitable Lyapunov-Krasovskii functional containing some novel triple integral terms with sufficient information about the actual sampling pattern. Based on the above discussion, the theory of time-delay systems can be divided into two classes: delay-independent control and delay-dependent control. To the best of our knowledge, delay-dependent stabilization condition gives less conservative result than the delay-independent one as it makes full use of information of the system. Specifically, systems with delay are of significant interest not only for their applicability in practice but also for their interesting theoretical properties. This is motivated by the need for systematic approach to investigate switched systems with delay.

On the other hand, the switching between the controller and the subsystem of switched systems is synchronous in the ideal case. In fact, the asynchronous phenomenon often occurs in practical industrial systems. For instance, when the system and the controller communicate via a communication channel and the current subsystem is switched to the next one, it is necessary to take some time to identify the active subsystem and then switch the controller from the current one to the corresponding subsystem, further causing asynchronous switching. With the great development of switched systems, the asynchronous control problem for switched systems, which is quite practical and energy efficient, has received increasing attention. In the past few years, it is noted that some valid results have appeared in studying nonlinear switched systems under asynchronous switching [26-29]. Specifically, [30] investigated the problem of output tracking control for switched systems with time-varying delay under asynchronous switching. Moreover, based on the dwell time approach, some sufficient conditions of exponential stabilization for a given switched system and a tracking error system were proposed in terms of linear matrix inequalities 
(LMIs). Due to the switching instants of the controllers lagging behind those of the subsystems, [31] dealt with the problem of stabilization for a class of switched delay systems with polytopic type uncertainties under asynchronous switching, and the running time was divided into two parts: matched periods $\left[t_{k}+\tau_{d}, t_{k+1}\right), k=1,2, \ldots$ and mismatched periods $\left[t_{k}, t_{k}+\tau_{d}\right), k=1,2, \ldots$ In addition, by constructing the parameter-dependent LyapunovKrasovskii functional and the average dwell time approach, the exponential stabilization problem for a class of nonlinear switched systems with mixed delays under asynchronous switching was investigated in [32]. In these papers mentioned above, the time delay is simple and no stochastic items are considered. Searching for delay-dependent mean-square stability criteria for nonlinear switched stochastic systems with interval time-varying delays is obviously more preferable and challenging.

Furthermore, it is well known that few results have been devoted to the stability of nonlinear switched stochastic systems with interval time-varying delay under asynchronous switching based on the average dwell time approach. This paper considers interval timevarying delay. It is natural to look for an alternative view to derive a less conservative condition for exponential stabilization of nonlinear switched stochastic systems under asynchronous switching. Moreover, we can hardly use the existing methods to investigate a stochastic switched system due to the impact of stochastic factor. This has motivated our present study on the following questions.

- Is it possible to find a delay-dependent multiple Lyapunov-Krasovskii functional that studies the matched periods and the mismatched periods of the nonlinear switched stochastic systems, respectively?

- Based on the average dwell time approach and Jense's inequality, can we obtain a less conservative sufficient condition of mean-square exponential stabilization for nonlinear switched stochastic systems with interval time-varying delay under asynchronous switching?

- Can we design a mean-square exponentially stable feedback controller for switched nonlinear systems under asynchronous switching by the matrix deformation technique and Schur compensation?

The core of this paper is the further development of switched stochastic nonlinear systems with interval time-varying delay under asynchronous switching. Moreover, we have proposed a detailed study and solutions on the above issues. Compared with the existing results on switched systems, the main contributions of this paper can be summarized as follows. (i) We consider the actual situation. In fact, the system needs to take some time to identify the active subsystem, and then switch the controller from the current subsystem to the corresponding subsystem, further causing asynchronous switching. (ii) According to Lyapunov stability theory, the Lyapunov-Krasovskii functional constructed in this paper is time-delay-dependent and depends on the switching signal of the controller. At the same time, it is allowed to increase the running time of the active subsystem with mismatch controller. The established Lyapunov-Krasovskii functional facilitates the analysis of the proposed problem. (iii) By utilizing the matrix inequality technique and the average dwell time approach, delay-dependent sufficient conditions are given to guarantee mean-square exponential stabilization for nonlinear switched stochastic systems under asynchronous switching. Moreover, state feedback controllers and switching signal of the switched stochastic systems are designed simultaneously under asynchronous switching 
through special operations of matrices and Schur complement without resorting to additional constraints on the switching signal.

The remainder of this paper is organized as follows. In Sect. 2, the problem description and preliminaries and some necessary lemmas are presented. Section 3 is devoted to deriving the results on exponential stabilization for switching signals by the average dwell time approach and delay-dependent multi-Lyapunov-Krasovskii functional. Moreover, feedback controller of nonlinear switched stochastic systems with interval time-varying delay under asynchronous switching is designed, which is the main result of this paper. In Sect. 4, an example is given to illustrate the results. The paper is concluded in Sect. 5.

The notations used in this paper are fairly standard. $R^{n}$ denotes the $\mathrm{n}$-dimensional Euclidean space. $A^{T}$ denotes the transpose of $A$. The symbol $*$ is used to denote the corresponding transposed block matrix. Diag $\{\cdots\}$ is a block-diagonal matrix. $I$ represents the identity matrix in the block matrix, and 0 represents a zero matrix with appropriate dimensions. The notation $P>0$ indicates that $P$ is a real symmetric and positive definite matrix, and $\lambda_{\min }\left(\lambda_{\max }\right)$ is the minimum (maximum) eigenvalue of $P$.

\section{Preliminaries}

Consider the following switched stochastic nonlinear systems with interval time-varying delay:

$$
\begin{aligned}
d x(t)= & {\left[A_{1 \sigma(t)} x(t)+A_{2 \sigma(t)} x(t-h(t))+B_{\sigma(t)} u(t)\right.} \\
& \left.+C_{\sigma(t)} f_{\sigma(t)}(t, x(t), x(t-h(t)))\right] d t+D_{\sigma(t)} x(t) d \omega(t), \\
x(s)= & \phi(s), \quad s \in\left[-h_{M}, 0\right],
\end{aligned}
$$

where $x(t) \in R^{n}$ and $u(t) \in R^{m}$ are, respectively, the state vector and the control input of switched systems. $\phi(s) \in R^{n}$ is the initial condition and $f_{\sigma(t)}(\cdot)$ are nonlinear functions. $\sigma(t):[0, \infty] \rightarrow M=\{1,2, \ldots, n\}$ is the switching signal. Specifically, denote $\Sigma$ : $\left\{\left(t_{0}, \sigma\left(t_{0}\right)\right), \ldots,\left(t_{k}, \sigma\left(t_{k}\right)\right), \ldots, k=0,1,2, \ldots\right\}$, where $0=t_{0}<t_{1}<t_{2}<\cdots<t_{k}<\cdots$, in which $t_{0}$ is the initial switching instant, $t_{k}$ denotes the $k t h$ switching instant. For $\sigma\left(t_{k}\right)=i$, $A_{1 i}, A_{2 i}, B_{i}, C_{i}, D_{i}$ are constant matrices with appropriate dimensions. In this article, we assume that the delay function $h(t)$ is interval time-varying and satisfies

$$
h_{m} \leq d(t) \leq h_{M}, \quad \dot{h}(t) \leq h<1 .
$$

$\omega(t)$ is a one-dimensional Brownian motion on a probability space $(\Omega, \mathcal{F}, \mathcal{P})$ and it satisfies the following cases:

$$
E\{d \omega(t)\}=0, \quad E\left\{d \omega^{2}(t)\right\}=d t .
$$

$f_{i}(t, x(t), x(t-h(t)))$ are nonlinear perturbation functions, which satisfy the following condition:

$$
\begin{aligned}
& f_{i}^{T}(t, x(t), x(t-h(t))) f_{i}(t, x(t), x(t-h(t))) \\
& \quad \leq x^{T}(t) V_{i}^{T} V_{i} x(t)+x^{T}(t-h(t)) \Lambda_{i}^{T} \Lambda_{i} x(t-h(t)),
\end{aligned}
$$


where $V_{i}$ and $\Lambda_{i}$ are known constant matrices. Note that the assumption on the nonlinear perturbations is widely applicable in practice and considered by many researchers. When the controller synchronizes with the switching subsystem, the state feedback controller is often designed as

$$
u(t)=K_{\sigma(t)} x(t)
$$

where $K_{i}, i \in M$, denotes the feedback gain matrix.

In practice, since it inevitably takes some time to identify the system modes and apply the matched controllers, the switching instants of the controllers lag behind those of the subsystems. At this point, we consider the state feedback given by

$$
u(t)=K_{\sigma\left(t-\tau_{d}\right)} x(t)
$$

where $\tau_{d}$ is a known constant.

Remark 1 In this paper, $\tau_{d}$ represents the period that the switching instants of the controller lag behind those of the system. Specifically, the running time of switched system is divided into two parts: matched periods $\left[t_{k}+\tau_{d}, t_{k+1}\right), k=1,2, \ldots$, and mismatched periods $\left[t_{k}, t_{k}+\tau_{d}\right), k=1,2, \ldots$. Correspondingly, we suppose that the $j$ th subsystem is activated at the switching instant $t_{k-1}$, and the $i$ th subsystem is activated at the switching instant $t_{k}$, then the corresponding switching controllers are activated at the switching $t_{k-1}+\tau_{d}$ and $t_{k}+\tau_{d}$, respectively.

The closed loop system of system (1) in the interval $\left[t_{k}, t_{k+1}\right)$ can be represented as:

$$
\begin{aligned}
d x(t)= & {\left[\bar{A}_{1 i j} x(t) x(t)+A_{2 i} x(t-h(t))+C_{i} f_{i}(t, x(t), x(t-h(t)))\right] d t } \\
& +D_{i} x(t) d \omega(t), \quad \forall t \in\left[t_{k}, t_{k}+\tau_{d}\right) ; \text { mismatched periods } \\
d x(t)= & {\left[\bar{A}_{1 i} x(t) x(t)+A_{2 i} x(t-h(t))+C_{i} f_{i}(t, x(t), x(t-h(t)))\right] d t } \\
& +D_{i} x(t) d \omega(t), \quad \forall t \in\left[t_{k}+\tau_{d}, t_{k+1}\right) ; \text { matched periods, }
\end{aligned}
$$

where $\bar{A}_{1 i j}=A_{1 i}+B_{i} K_{j}, \bar{A}_{1 i}=A_{1 i}+B_{i} K_{i}$.

Definition 1 ([28]) The equilibrium $x^{*}=0$ of the closed-loop system (7) is said to be mean-square exponentially stable under switching signal $\sigma(t)$ if the solution $x(t)$ of system satisfies

$$
E\left\{\|x(t)\|^{2}\right\} \leq k \sup _{-h_{M} \leq \theta \leq 0} E\left\{\left\|x\left(t_{0}+\theta\right)\right\|^{2}\right\} e^{-\alpha\left(t-t_{0}\right)}, \quad \forall t \geq t_{0}
$$

for constants $k \geq 1, \alpha>0$.

Definition 2 ([33]) For any $T_{2}>T_{1} \geq 0$, let $N_{\sigma}\left(T_{1}, T_{2}\right)$ denote the switching number of $\sigma(t)$ on an interval $\left(T_{1}, T_{2}\right)$. If

$$
N_{\sigma}\left(T_{1}, T_{2}\right) \geq N_{0}+\left(T_{2}-T_{1}\right) / \tau_{\alpha}
$$


holds for given $N_{0} \geq 0, \tau_{\alpha} \geq 0$, then the constant $\tau_{\alpha}$ is called the average dwell time and $N_{0}$ is the chatter bound. Without loss of generality, we choose $N_{0}=0$ in this paper.

Lemma 1 (Schur complement) For a given matrix $\left(\begin{array}{cc}S_{11} & S_{12} \\ * & S_{22}\end{array}\right)$ with $S_{11}=S_{11}^{T}, S_{22}=S_{22}^{T}$, then the following conditions are equivalent:

(1) $S<0$,

(2) $S_{11}<0, S_{22}-S_{12}^{T} S_{11}^{-1} S_{12}<0$,

(3) $S_{22}<0, S_{11}-S_{12} S_{22}^{-1} S_{12}^{T}<0$.

Lemma 2 (Jensen's inequality) For any symmetric and positive definite constant matrix $G \in R^{l \times l}$, scalars $\alpha$ and $\beta: \beta<\alpha$, vector function $x:[\beta, \alpha] \rightarrow R^{l}$ such that the integration concerned are well defined, then

$$
-\int_{\beta}^{\alpha} x^{T}(s) G x(s) d s \leq-\frac{1}{\alpha-\beta}\left(\int_{\beta}^{\alpha} x(s) d s\right)^{T} G\left(\int_{\beta}^{\alpha} x(s) d s\right) .
$$

\section{Main results}

In this section, based on the Lyapunov stability theory, a new piecewise multi-LyapunovKrasovskii functional dependent on the size of time delay is constructed. Moreover, we give sufficient conditions for the mean-square exponential stabilization of system (7) by the average dwell time approach and Jensen's inequality. In addition, the state feedback controllers of nonlinear switched systems are designed under asynchronous switching.

Theorem 1 For given positive constants $\alpha, \beta, h$, and $\mu \geq 1$, if there exist symmetric and positive definite matrices $P_{i}, Q_{1 i}, Q_{2 i}, Q_{3 i}, R_{1 i}, R_{2 i}$ such that the following matrix inequalities hold:

$$
\begin{aligned}
& P_{i} \leq \mu P_{j}, \quad Q_{1 i} \leq \mu Q_{1 j}, \quad Q_{2 i} \leq \mu Q_{2 j}, \\
& Q_{3 i} \leq \mu Q_{3 j}, \quad R_{1 i} \leq \mu R_{1 j}, \\
& \Xi_{i}=\left(\begin{array}{ccccccc}
\phi_{11}^{i} & P_{i} A_{2 i} & 0 & 0 & P_{i} C_{i} & 0 & 0 \\
* & \phi_{22}^{i} & 0 & 0 & 0 & 0 & 0 \\
* & * & \phi_{33}^{i} & 0 & 0 & 0 & 0 \\
* & * & * & \phi_{44}^{i} & 0 & 0 & 0 \\
* & * & * & * & -I & 0 & 0 \\
* & * & * & * & * & \phi_{66}^{i} & 0 \\
* & * & * & * & * & * & \phi_{77}^{i}
\end{array}\right) \\
& \Omega_{i}=\left(\begin{array}{ccccccc}
\varphi_{11}^{i} & P_{i} A_{2 i} & 0 & 0 & P_{i} C_{i} & 0 & 0 \\
* & \varphi_{22}^{i} & 0 & 0 & 0 & 0 & 0 \\
* & * & -Q_{2 i} & 0 & 0 & 0 & 0 \\
* & * & * & -Q_{3 i} & 0 & 0 & 0 \\
* & * & * & * & -I & 0 & 0 \\
* & * & * & * & * & -R_{1 i} & 0 \\
* & * & * & * & * & * & -R_{2 i}
\end{array}\right)<0,
\end{aligned}
$$


where

$$
\begin{aligned}
& \phi_{11}^{i}=\bar{A}_{1 i}^{T} P_{i}+P_{i} \bar{A}_{1 i}+Q_{1 i}+Q_{2 i}+Q_{3 i}+h_{m}^{2} R_{1 i}+h_{M}^{2} R_{2 i}+\alpha P_{i}+V_{i}^{T} V_{i}, \\
& \phi_{22}^{i}=\Lambda_{i}^{T} \Lambda_{i}-(1-h) e^{-\alpha h_{M}} Q_{1 i}, \quad \phi_{33}^{i}=-e^{-\alpha h_{m}} Q_{2 i}, \\
& \phi_{44}^{i}=-e^{-\alpha h_{M}} Q_{3 i}, \quad \phi_{66}^{i}=-e^{-\alpha h_{m}} R_{1 i}, \quad \phi_{77}^{i}=-e^{-\alpha h_{M}} R_{2 i} . \\
& \varphi_{11}^{i}=\bar{A}_{1 i j}^{T} P_{i}+P_{i} \bar{A}_{1 i j}+Q_{1 i}+Q_{2 i}+Q_{3 i}+h_{m}^{2} R_{1 i}+h_{M}^{2} R_{2 i}-\beta P_{i}+V_{i}^{T} V_{i}, \\
& \varphi_{22}^{i}=\Lambda_{i}^{T} \Lambda_{i}-(1-h) Q_{1 i} .
\end{aligned}
$$

If the average dwell time of the switching signal $\sigma(t)$ satisfies

$$
\tau_{a}>\tau_{a}^{*}=\frac{\ln \mu+(\alpha+\beta) \tau_{d}}{\alpha},
$$

then the closed-loop system (7) is mean-square exponentially stabilizable under arbitrary switching signal for the feedback control (6).

Proof When $t \in\left[t_{k}+\tau_{d}, t_{k+1}\right), \sigma\left(t_{k}\right)=i \in M$, switched systems run in matched periods. The closed-loop system (7) is active within the $i$ th subsystem, and the corresponding $i$ th switching controller is also activated. We choose the Lyapunov-Krasovskii functional candidate as follows:

$$
\begin{aligned}
V_{1 \sigma(t)}(t)= & x^{T}(t) P_{\sigma(t)} x(t)+\int_{t-h(t)}^{t} e^{\alpha(s-t)} x^{T}(s) Q_{1 \sigma(t)} x(s) d s \\
& +\int_{t-h_{m}}^{t} e^{\alpha(s-t)} x^{T}(s) Q_{2 \sigma(t)} x(s) d s \\
& +\int_{t-h_{M}}^{t} e^{\alpha(s-t)} x^{T}(s) Q_{3 \sigma(t)} x(s) d s \\
& +h_{m} \int_{-h_{m}}^{0} \int_{t+\theta}^{t} e^{\alpha(s-t)} x^{T}(s) R_{1 \sigma(t)} x(s) d s d \theta \\
& +h_{M} \int_{-h_{M}}^{0} \int_{t+\theta}^{t} e^{\alpha(s-t)} x^{T}(s) R_{2 \sigma(t)} x(s) d s d \theta .
\end{aligned}
$$

According to Itô's differential formula, the stochastic differential is

$$
d V_{1 i}(t)=\mathcal{L} V_{1 i} d t+2 x^{T}(t) P_{i} D_{i} x(t) d \omega(t)
$$

with the infinitesimal operator

$$
\begin{aligned}
\mathcal{L} V_{1 i}= & 2 x^{T}(t) P_{i}\left[\bar{A}_{1 i} x(t) x(t)+A_{2 i} x(t-h(t))+C_{i} f_{i}(t, x(t), x(t-h(t)))\right] \\
& +x^{T}(t) Q_{1 i} x(t)-(1-\dot{h}(t)) e^{-\alpha h(t)} x^{T}(t-h(t)) Q_{1 i} x(t-h(t)) \\
& +x^{T}(t) D_{i}^{T} P_{i} D_{i} x(t)+x^{T}(t) Q_{2 i} x(t)-e^{-\alpha h_{m}} x^{T}\left(t-h_{m}\right) Q_{2 i} x\left(t-h_{m}\right) \\
& +x^{T}(t) Q_{3 i} x(t)-e^{-\alpha h_{M}} x^{T}\left(t-h_{M}\right) Q_{3 i} x\left(t-h_{M}\right)+h_{m}^{2} x^{T}(t) R_{1 i} x(t) \\
& -h_{m} \int_{-h_{m}}^{0} e^{\alpha \theta} x^{T}(t+\theta) R_{1 i} x(t+\theta) d \theta+h_{M}^{2} x^{T}(t) R_{2 i} x(t)
\end{aligned}
$$




$$
\begin{aligned}
& -\alpha \int_{t-h_{m}}^{t} e^{\alpha(s-t)} x^{T}(s) Q_{2 i} x(s) d s-\alpha \int_{t-h_{M}}^{t} e^{\alpha(s-t)} x^{T}(s) Q_{3 i} x(s) d s \\
& -\alpha \int_{-h_{m}}^{0} \int_{t+\theta}^{t} e^{\alpha(s-t)} x^{T}(s) R_{1 i} x(s) d \theta \\
& -\alpha \int_{-h_{M}}^{0} \int_{t+\theta}^{t} e^{\alpha(s-t)} x^{T}(s) R_{2 i} x(s) d \theta \\
& -h_{M} \int_{-h_{M}}^{0} e^{\alpha \theta} x^{T}(t+\theta) R_{2 i} x(t+\theta) d \theta-\alpha \int_{t-h(t)}^{t} e^{\alpha(s-t)} x^{T}(s) Q_{1 i} x(s) d s \\
& \leq x^{T}(t)\left[P_{i} \bar{A}_{1 i}+\bar{A}_{1 i}^{T} P_{i}+Q_{1 i}+Q_{2 i}+Q_{3 i}+h_{m}^{2} R_{1 i}+h_{M}^{2} R_{2 i}+D_{i}^{T} P_{i} D_{i}\right] x(t) \\
& -\alpha \int_{-h_{m}}^{0} \int_{t+\theta}^{t} e^{\alpha(s-t)} x^{T}(s) R_{1 i} x(s) d s \\
& -\alpha \int_{-h_{M}}^{0} \int_{t+\theta}^{t} e^{\alpha(s-t)} x^{T}(s) R_{2 i} x(s) d s-\alpha \int_{t-h(t)}^{t} e^{\alpha(s-t)} x^{T}(s) Q_{1 i} x(s) d s \\
& -h_{m} \int_{t-h_{m}}^{t} e^{-\alpha h_{m}} x^{T}(s) R_{1 i} x(s) d s \\
& -\alpha \int_{t-h_{m}}^{t} e^{\alpha(s-t)} x^{T}(s) Q_{2 i} x(s) d s \\
& -h_{M} \int_{t-h_{M}}^{t} e^{-\alpha h_{M}} x^{T}(s) R_{2 i} x(s) d s \\
& -\alpha \int_{t-h_{M}}^{t} e^{\alpha(s-t)} x^{T}(s) Q_{3 i} x(s) d s-e^{-\alpha h_{m}} x^{T}\left(t-h_{m}\right) Q_{2 i} x\left(t-h_{m}\right) \\
& +x^{T}(t) P_{i} A_{2 i} x(t-h(t))+x^{T}(t-h(t)) A_{2 i}^{T} P_{i} x(t) \\
& +f_{i}^{T}(t, x(t), x(t-h(t))) C_{i}^{T} P_{i} x(t) e^{-\alpha h_{M}} x^{T}\left(t-h_{M}\right) Q_{3 i} x\left(t-h_{M}\right) \\
& -(1-h) e^{-\alpha h_{M}} x^{T}(t-h(t)) Q_{1 i} x(t-h(t)) \\
& +x^{T}(t) P_{i} C_{i} f_{i}(t, x(t), x(t-h(t))) \text {. }
\end{aligned}
$$

Inequality (4) can be written as follows:

$$
\begin{aligned}
& x^{T}(t) V_{i}^{T} V_{i} x(t)+x^{T}(t-h(t)) \Lambda_{i}^{T} \Lambda_{i} x(t-h(t)) \\
& \quad-f_{i}^{T}(t, x(t), x(t-h(t))) f_{i}(t, x(t), x(t-h(t))) \geq 0 .
\end{aligned}
$$

By using Lemma 2, we get

$$
\begin{aligned}
& -h_{m} \int_{t-h_{m}}^{t} e^{-\alpha h_{m}} x^{T}(s) R_{1 i} x(s) d s \\
& \leq-e^{-\alpha h_{m}}\left(\int_{t-h_{m}}^{t} x(s) d s\right)^{T} R_{1 i}\left(\int_{t-h_{m}}^{t} x(s) d s\right), \\
& -h_{M} \int_{t-h_{M}}^{t} e^{-\alpha h_{M}} x^{T}(s) R_{2 i} x(s) d s \\
& \leq-e^{-\alpha h_{M}}\left(\int_{t-h_{M}}^{t} x(s) d s\right)^{T} R_{2 i}\left(\int_{t-h_{M}}^{t} x(s) d s\right) .
\end{aligned}
$$


From (16), (17), and (18) we have

$$
\begin{aligned}
\mathcal{L} V_{1 i}+\alpha V_{1 i} \leq & x^{T}(t)\left[P_{i} \bar{A}_{1 i}+\bar{A}_{1 i}^{T} P_{i}+Q_{1 i}+Q_{2 i}+Q_{3 i}\right. \\
& \left.+\alpha P_{i}+h_{m}^{2} R_{1 i}+h_{M}^{2} R_{2 i}+D_{i}^{T} P_{i} D_{i}+V_{i}^{T} V_{i}\right] x(t) \\
& +x^{T}(t) P_{i} C_{i} f_{i}(t, x(t), x(t-h(t))) \\
& +f_{i}^{T}(t, x(t), x(t-h(t))) C_{i}^{T} P_{i} x(t) \\
& -f_{i}^{T}(t, x(t), x(t-h(t))) f_{i}(t, x(t), x(t-h(t))) \\
& +x^{T}(t-h(t))\left(\Lambda_{i}^{T} \Lambda_{i}-(1-h) e^{-\alpha h_{M}} Q_{1 i}\right) x(t-h(t)) \\
& +x^{T}(t) P_{i} A_{2 i} x(t-h(t))+x^{T}(t-h(t)) A_{2 i}^{T} P_{i} x(t) \\
& -e^{-\alpha h_{m}}\left(\int_{t-h_{m}}^{t} x(s) d s\right)^{T} R_{1 i}\left(\int_{t-h_{m}}^{t} x(s) d s\right) \\
& -e^{-\alpha h_{m}} x^{T}\left(t-h_{m}\right) Q_{2 i} x\left(t-h_{m}\right) \\
& -e^{-\alpha h_{M}}\left(\int_{t-h_{M}}^{t} x(s) d s\right)^{T} R_{2 i}\left(\int_{t-h_{M}}^{t} x(s) d s\right) \\
& -e^{-\alpha h_{M}} x^{T}\left(t-h_{M}\right) Q_{3 i} x\left(t-h_{M}\right) .
\end{aligned}
$$

Let

$$
\begin{aligned}
\xi(t)= & \left(\begin{array}{llll}
x^{T}(t) & x^{T}(t-h(t)) & x^{T}\left(t-h_{m}\right) & x^{T}\left(t-h_{M}\right)
\end{array}\right. \\
& \left.f_{i}^{T}(t, x(t), x(t-h(t))) \quad\left(\int_{t-h_{m}}^{t} x(s) d s\right)^{T} \quad\left(\int_{t-h_{M}}^{t} x(s) d s\right)^{T}\right)^{T} .
\end{aligned}
$$

According to (19), we can obtain

$$
\mathcal{L} V_{1 i}+\alpha V_{1 i} \leq \xi^{T}(t) \Xi_{i} \xi(t)
$$

We can get

$$
\mathcal{L} V_{1 i} \leq-\alpha V_{1 i}
$$

Then, using (9) and (13), we have

$$
\begin{aligned}
d\left(e^{\alpha t} V_{1 i}\right) & =e^{\alpha t}\left[\alpha V_{1 i} d t+\mathcal{L} V_{1 i} d t+2 x^{T}(t) P_{i} D_{i} x(t) d \omega(t)\right] \\
& \leq e^{\alpha t}\left[\alpha V_{1 i} d t-\alpha V_{1 i} d t+2 x^{T}(t) P_{i} D_{i} x(t) d \omega(t)\right] \\
& =2 e^{\alpha t} x^{T}(t) P_{i} D_{i} x(t) d \omega(t) .
\end{aligned}
$$

When $t \in\left[t_{k}, t_{k}+\tau_{d}\right)$, switched systems run in mismatched periods. The closed-loop system (7) is active within the $i$ th subsystem and the corresponding $j$ th switching controller is also activated. We choose the Lyapunov-Krasovskii functional candidate as follows:

$$
V_{2 \sigma(t)}(t)=x^{T}(t) P_{\sigma(t)} x(t)+\int_{t-h(t)}^{t} e^{\beta(t-s)} x^{T}(s) Q_{1 \sigma(t)} x(s) d s
$$




$$
\begin{aligned}
& +\int_{t-h_{m}}^{t} e^{\beta(t-s)} x^{T}(s) Q_{2 \sigma(t)} x(s) d s \\
& +\int_{t-h_{M}}^{t} e^{\beta(t-s)} x^{T}(s) Q_{3 \sigma(t)} x(s) d s \\
& +h_{m} \int_{-h_{m}}^{0} \int_{t+\theta}^{t} e^{\beta(t-s)} x^{T}(s) R_{1 \sigma(t)} x(s) d s d \theta \\
& +h_{M} \int_{-h_{M}}^{0} \int_{t+\theta}^{t} e^{\beta(t-s)} x^{T}(s) R_{2 \sigma(t)} x(s) d s d \theta
\end{aligned}
$$

According to Itô's differential formula, we have

$$
\begin{aligned}
& \mathcal{L} V_{2 i}=2 x^{T}(t) P_{i}\left[\bar{A}_{1 i j} x(t) x(t)+A_{2 i} x(t-h(t))+C_{i} f_{i}(t, x(t), x(t-h(t)))\right] \\
& +x^{T}(t) Q_{1 i} x(t)+x^{T}(t) D_{i}^{T} P_{i} D_{i} x(t)-+x^{T}(t) Q_{2 i} x(t) \\
& -(1-\dot{h}(t)) e^{\beta h(t)} x^{T}(t-h(t)) Q_{1 i} x(t-h(t)) \\
& -e^{\beta h_{m}} x^{T}\left(t-h_{m}\right) Q_{2 i} x\left(t-h_{m}\right)+h_{m}^{2} x^{T}(t) R_{1 i} x(t) \\
& +x^{T}(t) Q_{3 i} x(t)-e^{\beta h_{M}} x^{T}\left(t-h_{M}\right) Q_{3 i} x\left(t-h_{M}\right) \\
& -h_{m} \int_{-h_{m}}^{0} e^{-\beta \theta} x^{T}(t+\theta) R_{1 i} x(t+\theta) d \theta+h_{M}^{2} x^{T}(t) R_{2 i} x(t) \\
& -h_{M} \int_{-h_{M}}^{0} e^{-\beta \theta} x^{T}(t+\theta) R_{2 i} x(t+\theta) d \theta \\
& +\beta \int_{t-h(t)}^{t} e^{\beta(t-s)} x^{T}(s) Q_{1 i} x(s) d s \\
& +\beta \int_{t-h_{m}}^{t} e^{\beta(t-s)} x^{T}(s) Q_{2 i} x(s) d s \\
& +\beta \int_{t-h_{M}}^{t} e^{\beta(t-s)} x^{T}(s) Q_{3 i} x(s) d s \\
& +\beta \int_{-h_{m}}^{0} \int_{t+\theta}^{t} e^{\beta(t-s)} x^{T}(s) R_{1 i} x(s) d s d \theta \\
& +\beta \int_{-h_{M}}^{0} \int_{t+\theta}^{t} e^{\beta(t-s)} x^{T}(s) R_{2 i} x(s) d s d \theta \\
& \leq x^{T}(t)\left[P_{i} \bar{A}_{1 i j}+\bar{A}_{1 i j}^{T} P_{i}+Q_{1 i}+Q_{2 i}+Q_{3 i}+h_{m}^{2} R_{1 i}+h_{M}^{2} R_{2 i}\right. \\
& \left.+D_{i}^{T} P_{i} D_{i}\right] x(t)-(1-h) x^{T}(t-h(t)) Q_{1 i} x(t-h(t)) \\
& +x^{T}(t) P_{i} C_{i} f_{i}(t, x(t), x(t-h(t)))+f_{i}^{T}(t, x(t), x(t-h(t))) C_{i}^{T} P_{i} x(t) \\
& -x^{T}\left(t-h_{M}\right) Q_{3 i} x\left(t-h_{M}\right)+\beta \int_{-h_{m}}^{0} \int_{t+\theta}^{t} e^{\beta(t-s)} x^{T}(s) R_{1 i} x(s) d s d \theta \\
& +x^{T}(t-h(t)) A_{2 i}^{T} P_{i} x(t)+\beta \int_{t-h(t)}^{t} e^{\beta(t-s)} x^{T}(s) Q_{1 i} x(s) d s \\
& -h_{m} \int_{t-h_{m}}^{t} x^{T}(s) R_{1 i} x(s) d s+\beta \int_{t-h_{m}}^{t} e^{\alpha(t-s)} x^{T}(s) Q_{2 i} x(s) d s
\end{aligned}
$$




$$
\begin{aligned}
& -h_{M} \int_{t-h_{M}}^{t} x^{T}(s) R_{2 i} x(s) d s+\beta \int_{t-h_{M}}^{t} e^{\beta(t-s)} x^{T}(s) Q_{3 i} x(s) d s \\
& -x^{T}\left(t-h_{m}\right) Q_{2 i} x\left(t-h_{m}\right)+\beta \int_{-h_{M}}^{0} \int_{t+\theta}^{t} e^{\beta(t-s)} x^{T}(s) R_{2 i} x(s) d s d \theta \\
& +x^{T}(t) P_{i} A_{2 i} x(t-h(t)) .
\end{aligned}
$$

Following the similar way, we have

$$
\begin{aligned}
\mathcal{L} V_{2 i}-\beta V_{2 i} \leq & x^{T}(t)\left[P_{i} \bar{A}_{1 i j}+\bar{A}_{1 i j}^{T} P_{i}+Q_{1 i}+Q_{2 i}+Q_{3 i}-\beta P_{i}+h_{m}^{2} R_{1 i}\right. \\
& \left.+h_{M}^{2} R_{2 i}+D_{i}^{T} P_{i} D_{i}+V_{i}^{T} V_{i}\right] x(t)+x^{T}(t-h(t)) A_{2 i}^{T} P_{i} x(t) \\
& +x^{T}(t) P_{i} C_{i} f_{i}(t, x(t), x(t-h(t)))+x^{T}(t) P_{i} A_{2 i} x(t-h(t)) \\
& +f_{i}^{T}(t, x(t), x(t-h(t))) C_{i}^{T} P_{i} x(t) \\
& -f_{i}^{T}(t, x(t), x(t-h(t))) f_{i}(t, x(t), x(t-h(t))) \\
& +x^{T}(t-h(t))\left(\Lambda_{i}^{T} \Lambda_{i}-(1-h) Q_{1 i}\right) x(t-h(t)) \\
& -x^{T}\left(t-h_{m}\right) Q_{2 i} x\left(t-h_{m}\right)-x^{T}\left(t-h_{M}\right) Q_{3 i} x\left(t-h_{M}\right) \\
& -\left(\int_{t-h_{m}}^{t} x(s) d s\right)^{T} R_{1 i}\left(\int_{t-h_{m}}^{t} x(s) d s\right) \\
& -\left(\int_{t-h_{M}}^{t} x(s) d s\right)^{T} R_{2 i}\left(\int_{t-h_{M}}^{t} x(s) d s\right) .
\end{aligned}
$$

According to (12), we have

$$
\mathcal{L} V_{2 i}-\beta V_{2 i} \leq \xi^{T}(t) \Omega_{i} \xi(t)
$$

Then

$$
\mathcal{L} V_{2 i} \leq \beta V_{2 i}
$$

we can get

$$
\begin{aligned}
d\left(e^{-\beta t} V_{2 i}\right) & =e^{-\beta t}\left[-\beta V_{2 i} d t+\mathcal{L} V_{2 i} d t+2 x^{T}(t) P_{i} D_{i} x(t) d \omega(t)\right] \\
& \leq e^{-\beta t}\left[-\beta V_{2 i} d t+\beta V_{2 i} d t+2 x^{T}(t) P_{i} D_{i} x(t) d \omega(t)\right] \\
& =2 e^{-\beta t} x^{T}(t) P_{i} D_{i} x(t) d \omega(t) .
\end{aligned}
$$

By recalling (2), we have

$$
\begin{aligned}
& \int_{t-h(t)}^{t} e^{\alpha(s-t)} x^{T}(s) Q_{1 i} x(s) d s+\int_{t-h_{m}}^{t} e^{\alpha(s-t)} x^{T}(s) Q_{2 i} x(s) d s \\
& \quad+\int_{t-h_{M}}^{t} e^{\alpha(s-t)} x^{T}(s) Q_{3 i} x(s) d s+\int_{-h_{m}}^{0} \int_{t+\theta}^{t} e^{\alpha(s-t)} x^{T}(s) R_{1 i} x(s) d s d \theta \\
& \quad+\int_{-h_{M}}^{0} \int_{t+\theta}^{t} e^{\alpha(s-t)} x^{T}(s) R_{2 i} x(s) d s d \theta
\end{aligned}
$$




$$
\begin{aligned}
\leq & \int_{t-h(t)}^{t} x^{T}(s) Q_{i} x(s) d s+\int_{t-h_{m}}^{t} x^{T}(s) Q_{2 i} x(s) d s \\
& +\int_{t-h_{M}}^{t} x^{t}(s) Q_{3 i} x(s) d s+\int_{-h_{m}}^{0} \int_{t+\theta}^{t} x^{T}(s) R_{1 i} x(s) d s d \theta \\
& +\int_{-h_{M}}^{0} \int_{t+\theta}^{T} x^{T}(s) R_{2 i} x(s) d s d \theta \\
\leq & \int_{t-h(t)}^{t} e^{\beta(t-s)} x^{T}(s) Q_{1 i} x(s) d s+\int_{t-h_{m}}^{t} e^{\beta(t-s)} x^{T}(s) Q_{2 i} x(s) d s \\
& +\int_{t-h_{M}}^{t} e^{\beta(t-s)} x^{T}(s) Q_{3 i} x(s) d s+\int_{-h_{m}}^{0} \int_{t+\theta}^{t} e^{\beta(t-s)} x^{T}(s) R_{1 i} x(s) d s d \theta \\
& +\int_{-h_{M}}^{0} \int_{t+\theta}^{t} e^{\beta(t-s)} x^{T}(s) R_{2 i} x(s) d s d \theta .
\end{aligned}
$$

Therefore,

$$
V_{1 i}(t) \leq V_{2 i}(t)
$$

Considering the whole interval $\left[t_{0}, t\right)$, the Lyapunov-Krasovskii functional $V(t)$ is expressed as

$$
\begin{aligned}
& V_{1 \sigma(t)}(t) \quad \forall t \in\left[t_{k}+\tau_{d}, t_{k+1}\right), k=0,1,2, \ldots ; \\
& V_{2 \sigma(t)}(t) \quad \forall t \in\left[t_{k}, t_{k}+\tau_{d}\right), k=0,1,2, \ldots
\end{aligned}
$$

When $t \in\left[t_{k}+\tau_{d}, t_{k+1}\right)$, integrating both sides of (22) from $t_{k}+\tau_{d}$ to $t$ and taking expectation, we have

$$
\begin{aligned}
E\{V(t)\} & =E\left\{V_{1 i}(t)\right\} \leq e^{-\alpha\left(t-\left(t_{k}+\tau_{d}\right)\right)} E\left\{V_{1 i}\left(\left(t_{k}+\tau_{d}\right)^{+}\right)\right\} \\
& \leq e^{-\alpha\left(t-\left(t_{k}+\tau_{d}\right)\right)} E\left\{V_{2 i}\left(\left(t_{k}+\tau_{d}\right)^{-}\right)\right\} \\
& \leq e^{-\alpha\left(t-\left(t_{k}+\tau_{d}\right)\right)} e^{\beta \tau_{d}} E\left\{V_{2 i}\left(\left(t_{k}\right)^{+}\right)\right\} \\
& \leq \mu e^{-\alpha\left(t-\left(t_{k}+\tau_{d}\right)\right)} e^{\beta \tau_{d}} E\left\{V_{1 i}\left(\left(t_{k}\right)^{+}\right)\right\} \\
& \leq \ldots \\
& \leq \mu^{k} e^{(k+1) \beta \tau_{d}} e^{-\alpha\left(t-t_{0}-(k+1) \tau_{d}\right)} E\left\{V\left(t_{0}\right)\right\} \\
& \leq e^{(\alpha+\beta) \tau_{d}} e^{\left\{\left[\ln \mu+(\alpha+\beta) \tau_{d}\right] / \tau_{a}-\alpha\right\}\left(t-t_{0}\right)} E\left\{V\left(t_{0}\right)\right\}
\end{aligned}
$$

When $t \in\left[t_{k}, t_{k}+\tau_{d}\right)$, integrating both sides of (28) and taking expectation, we obtain

$$
\begin{aligned}
E\{V(t)\} & =E\left\{V_{2 i}(t)\right\} \leq e^{\beta\left(t-\left(t_{k}\right)\right)} E\left\{V_{2 i}\left(\left(t_{k}\right)^{+}\right)\right\} \\
& \leq \mu e^{\beta\left(t-t_{k}\right)} E\left\{V_{1 i}\left(\left(t_{k}\right)^{-}\right)\right\} \\
& \leq \cdots \\
& \leq \mu^{k} e^{(k+1) \beta \tau_{d}} e^{-\alpha\left(t-t_{0}-(k+1) \tau_{d}\right)} E\left\{V\left(t_{0}\right)\right\} \\
& \leq e^{(\alpha+\beta) \tau_{d}} e^{\left\{\left[\ln \mu+(\alpha+\beta) \tau_{d}\right] / \tau_{a}-\alpha\right\}\left(t-t_{0}\right)} E\left\{V\left(t_{0}\right)\right\} .
\end{aligned}
$$


Notice from (14) and (23) that

$$
E\{V(t)\} \geq a E\left\{\|x(t)\|^{2}\right\}, \quad E\left\{V\left(t_{0}\right)\right\} \leq b \sup _{-h_{M} \leq \theta \leq 0} E\left\{\left\|x\left(t_{0}+\theta\right)\right\|^{2}\right\},
$$

where

$$
\begin{aligned}
a= & \min _{i \in M} \lambda_{\min }\left(P_{i}\right), \\
b= & \max _{i \in M} \lambda_{\max }\left(P_{i}\right)+h_{M} \max _{i \in M} \lambda_{\max }\left(Q_{1 i}\right)+h_{m} \max _{i \in M} \lambda_{\max }\left(Q_{2 i}\right) \\
& +h_{M} \max _{i \in M} \lambda_{\max }\left(Q_{3 i}\right)+\frac{h_{m}^{3}}{2} \max _{i \in M} \lambda_{\max }\left(R_{1 i}\right)+\frac{h_{M}^{3}}{2} \max _{i \in M} \lambda_{\max }\left(R_{2 i}\right) .
\end{aligned}
$$

Finally, we can get

$$
\begin{aligned}
E\left\{\|x(t)\|^{2}\right\} \leq & e^{(\alpha+\beta) \tau_{d}} \frac{b}{a} \sup _{-h_{M} \leq \theta \leq 0} E\left\{\left\|x\left(t_{0}+\theta\right)\right\|^{2}\right\} \\
& \times e^{\left\{\alpha-\left[\ln \mu+(\alpha+\beta) \tau_{d}\right] / \tau_{a}\right\}\left(t-t_{0}\right)} .
\end{aligned}
$$

By Definition 1, we know that the closed-loop system (7) is mean-square exponentially stabilizable. This completes the proof.

Theorem 2 For given positive constants $\alpha, \beta, h$, and $\mu \geq 1$, if there exist symmetric and positive definite matrices $X_{i}, S_{1 i}, S_{2 i}, S_{3 i}, T_{1 i}, T_{2 i}$ and any $Y_{i}$ such that the following matrix inequalities hold:

$$
\begin{aligned}
& X_{i} \leq \mu X_{j}, \quad S_{1 i} \leq \mu S_{1 j}, \quad S_{2 i} \leq \mu S_{2 j}, \\
& S_{3 i} \leq \mu S_{3 j}, \quad T_{1 i} \leq \mu T_{1 j}, \quad T_{2 i} \leq \mu T_{2 j}, \quad i, j \in M, i \neq j \\
& \left(\begin{array}{cc}
\Phi_{11}^{i} & \Phi_{12}^{i} \\
* & \Phi_{22}^{i}
\end{array}\right)<0, \\
& \left(\begin{array}{cc}
\Psi_{11}^{i} & \Phi_{12}^{i} \\
* & \Phi_{22}^{i}
\end{array}\right)<0,
\end{aligned}
$$

where

$$
\begin{aligned}
\Phi_{11}^{i} & =\left(\begin{array}{ccccccccc}
\hat{\phi}_{11}^{i} & \hat{\phi}_{12}^{i} & 0 & 0 & C_{i} & 0 & 0 & \hat{\phi}_{18}^{i} & 0 \\
* & \hat{\phi}_{22}^{i} & 0 & 0 & 0 & 0 & 0 & 0 & \hat{\phi}_{29}^{i} \\
* & * & \hat{\phi}_{33}^{i} & 0 & 0 & 0 & 0 & 0 & 0 \\
* & * & * & \hat{\phi}_{44}^{i} & 0 & 0 & 0 & 0 & 0 \\
* & * & * & * & -I & 0 & 0 & 0 & 0 \\
* & * & * & * & * & \hat{\phi}_{66}^{i} & 0 & 0 & 0 \\
* & * & * & * & * & * & \hat{\phi}_{77}^{i} & 0 & 0 \\
* & * & * & * & * & * & * & -I & 0 \\
* & * & * & * & * & * & * & * & -I
\end{array}\right), \\
\Phi_{12}^{i} & =\left(\begin{array}{ccccccc}
X_{i} D_{i}^{T} & X_{i} & X_{i} & X_{i} & h_{m} X_{i} & h_{M} X_{i} \\
\mathbf{0}_{8 \times 1} & \mathbf{0}_{8 \times 1} & \mathbf{0}_{8 \times 1} & \mathbf{0}_{8 \times 1} & \mathbf{0}_{8 \times 1} & \mathbf{0}_{8 \times 1}
\end{array}\right),
\end{aligned}
$$




$$
\begin{aligned}
& \Phi_{22}^{i}=\operatorname{diag}\left\{-X_{i},-S_{1 i},-S_{2 i},-S_{3 i},-T_{1 i},-T_{2 i}\right\} \text {, } \\
& \Psi_{11}^{i}=\left(\begin{array}{ccccccccc}
\hat{\varphi}_{11}^{i} & \hat{\phi}_{12}^{i} & 0 & 0 & C_{i} & 0 & 0 & \hat{\varphi}_{18}^{i} & 0 \\
* & \hat{\varphi}_{22}^{i} & 0 & 0 & 0 & 0 & 0 & 0 & \hat{\varphi}_{29}^{i} \\
* & * & \hat{\varphi}_{33}^{i} & 0 & 0 & 0 & 0 & 0 & 0 \\
* & * & * & \hat{\varphi}_{44}^{i} & 0 & 0 & 0 & 0 & 0 \\
* & * & * & * & -I & 0 & 0 & 0 & 0 \\
* & * & * & * & * & \hat{\varphi}_{66}^{i} & 0 & 0 & 0 \\
* & * & * & * & * & * & \hat{\varphi}_{77}^{i} & 0 & 0 \\
* & * & * & * & * & * & * & -I & 0 \\
* & * & * & * & * & * & * & * & -I
\end{array}\right) \\
& \hat{\phi}_{11}^{i}=A_{1 i} X_{i}+B_{i} K_{i} X_{i}+\left(A_{1 i} X_{i}+B_{i} K_{i} X_{i}\right)^{T}+\alpha X_{i}, \\
& \hat{\phi}_{22}^{i}=(1-h) e^{-\alpha h_{M}}\left(S_{1 i}-2 X_{i}\right), \quad \hat{\phi}_{12}^{i}=A_{2 i} X_{i}, \quad \hat{\phi}_{18}^{i}=X_{i} V_{i}^{T}, \\
& \hat{\phi}_{29}^{i}=X_{i} \Lambda_{i}^{T}, \quad \hat{\phi}_{33}^{i}=e^{-\alpha h_{m}}\left(S_{2 i}-2 X_{i}\right), \quad \hat{\phi}_{44}^{i}=e^{-\alpha h_{M}}\left(S_{3 i}-2 X_{i}\right), \\
& \hat{\phi}_{66}^{i}=e^{-\alpha h_{m}}\left(R_{1 i}-2 X_{i}\right), \quad \hat{\phi}_{77}^{i}=e^{-\alpha h_{M}}\left(R_{2 i}-2 X_{i}\right), \\
& \hat{\varphi}_{11}^{i}=A_{1 i} X_{i}+B_{i} K_{j} X_{i}+\left(A_{1 i} X_{i}+B_{i} K_{j} X_{i}\right)^{T}-\beta X_{i} \text {, } \\
& \hat{\varphi}_{22}^{i}=(1-h)\left(S_{1 i}-2 X_{i}\right), \quad \hat{\varphi}_{33}^{i}=S_{2 i}-2 X_{i}, \quad \hat{\varphi}_{44}^{i}=S_{3 i}-2 X_{i}, \\
& \hat{\phi}_{66}^{i}=T_{1 i}-2 X_{i}, \quad \hat{\phi}_{77}^{i}=T_{2 i}-2 X_{i},
\end{aligned}
$$

then system (1) is mean-square exponentially stabilizable for arbitrary switching signal with the average dwell time satisfying (13). In addition, the feedback controller can be designed by the following formula:

$$
K_{i}=Y_{i} X_{i}^{-1}, \quad i \in M
$$

Proof According to $S_{i}>0, T_{i}>0$, we have

$$
\begin{array}{ll}
\left(S_{p i}-X_{i}\right)^{T} S_{p i}{ }^{-1}\left(S_{p i}-X_{i}\right) \geq 0 \quad(p=1,2,3), \\
\left(T_{q i}-X_{i}\right)^{T} T_{q i}{ }^{-1}\left(T_{q i}-X_{i}\right) \geq 0 \quad(q=1,2) .
\end{array}
$$

Then the following inequality can be obtained:

$$
S_{p i}-2 X_{i} \geq-X_{i} S_{p i}{ }^{-1} X_{i}, \quad T_{q i}-2 X_{i} \geq-X_{i} T_{q i}{ }^{-1} X_{i} .
$$

Substituting (40) into (37) and multiplying both sides of (37) by $\operatorname{diag}\left\{X_{i}^{-1}, X_{i}^{-1}, X_{i}^{-1}, X_{i}^{-1}, I\right.$, $\left.X_{i}^{-1}, X_{i}^{-1}, I, I, I, I, I, I, I, I\right\}$, we can get the following inequality:

$$
\left(\begin{array}{cc}
\bar{\Phi}_{11}^{i} & \bar{\Phi}_{12}^{i} \\
* & \Phi_{22}^{i}
\end{array}\right)<0,
$$


where

$$
\begin{aligned}
& \bar{\Phi}_{11}^{i}=\left(\begin{array}{ccccccccc}
\tilde{\phi}_{11}^{i} & \tilde{\phi}_{12}^{i} & 0 & 0 & X_{i}^{-1} C_{i} & 0 & 0 & V_{i}^{T} & 0 \\
* & \tilde{\phi}_{22}^{i} & 0 & 0 & 0 & 0 & 0 & 0 & \Lambda_{i}^{T} \\
* & * & \tilde{\phi}_{33}^{i} & 0 & 0 & 0 & 0 & 0 & 0 \\
* & * & * & \tilde{\phi}_{44}^{i} & 0 & 0 & 0 & 0 & 0 \\
* & * & * & * & -I & 0 & 0 & 0 & 0 \\
* & * & * & * & * & \tilde{\phi}_{66}^{i} & 0 & 0 & 0 \\
* & * & * & * & * & * & \tilde{\phi}_{77}^{i} & 0 & 0 \\
* & * & * & * & * & * & * & -I & 0 \\
* & * & * & * & * & * & * & * & -I
\end{array}\right) \\
& \bar{\Phi}_{12}^{i}=\left(\begin{array}{cccccc}
D_{i}^{T} & I & I & I & h_{m} & h_{M} \\
\mathbf{0}_{8 \times 1} & \mathbf{0}_{8 \times 1} & \mathbf{0}_{8 \times 1} & \mathbf{0}_{8 \times 1} & \mathbf{0}_{8 \times 1} & \mathbf{0}_{8 \times 1}
\end{array}\right), \\
& \tilde{\phi}_{11}^{i}=X_{i}^{-1} A_{1 i}+X_{i}^{-1} B_{i} Y_{i} X_{i}^{-1}+\left(X_{i}^{-1} A_{1 i}+X_{i}^{-1} B_{i} Y_{i} X_{i}^{-1}\right)^{T}+\alpha X_{i}^{-1}, \\
& \tilde{\phi}_{12}^{i}=X_{i}^{-1} A_{2 i}, \quad \tilde{\phi}_{22}^{i}=-(1-h) e^{-\alpha h_{M}} S_{1 i}^{-1}, \quad \tilde{\phi}_{33}^{i}=-e^{-\alpha h_{m}} S_{2 i}^{-1}, \\
& \hat{\phi}_{29}^{i}=X_{i} \Lambda_{i}^{T}, \quad \hat{\phi}_{33}^{i}=e^{-\alpha h_{m}}\left(S_{2 i}-2 X_{i}\right), \quad \hat{\phi}_{44}^{i}=e^{-\alpha h_{M}}\left(S_{3 i}-2 X_{i}\right), \\
& \tilde{\phi}_{44}^{i}=-e^{-\alpha h_{M}} S_{3 i}^{-1}, \quad \bar{\phi}_{66}^{i}=-e^{-\alpha h_{m}} R_{1 i}^{-1}, \quad \tilde{\phi}_{77}^{i}=-e^{-\alpha h_{M}} R_{2 i}^{-1} .
\end{aligned}
$$

Then set

$$
\begin{aligned}
& Y_{i}=K_{i} X_{i}, \quad X_{i}^{-1}=P_{i}, \quad S_{1 i}{ }^{-1}=Q_{1 i}, \quad S_{2 i}{ }^{-1}=Q_{2 i}, \\
& S_{3 i}{ }^{-1}=Q_{3 i}, \quad T_{1 i}^{-1}=R_{1 i}, \quad T_{2 i}^{-1}=R_{2 i} .
\end{aligned}
$$

Using Schur complement in (41), it can be concluded that (12) holds. By the same method, (38) implies (13). Correspondingly, controller gains are given by (39). The proof is completed.

Remark 2 It is noticed that the Lyapunov-Krasovskii functional is delay-dependent in this paper. On the one hand, the important information of $h_{m}$ and $h_{M}$ is taken into full consideration, which may overcome the conservatism of quadratic mean-square exponential stability conditions for nonlinear switched stochastic systems with interval time-varying delay under asynchronous switching. On the other hand, the delay-dependent LyapunovKrasovskii functional is allowed to rise at both switching instants. However, the delaydependent Lyapunov-Krasovskii functional is decreasing on the entire interval and the mean-square exponential stabilization for nonlinear switched stochastic systems is also guaranteed.

Remark 3 [34] investigated the stabilization problem for a class of positive switched nonlinear systems under asynchronous switching. The author mainly focused on the study of positive switched systems in [34]. [35] addressed the problem of robust control for uncertain switched nonlinear systems with time delay under asynchronous switching. However, stochastic disturbance was not considered in [34] and [35]. The problem of robust reliable control for a class of uncertain stochastic switched nonlinear systems under asynchronous switching was investigated in [36], but the interval time-varying delay was not 
considered in [36] under asynchronous switching. In this paper, we consider stochastic disturbance and interval time-varying delay. Compared with [34-36], it is obvious that we have considered more external factors, and our switched systems model is more in line with engineering practice from the application level.

Remark 4 [18] obtained sufficient conditions with delay-dependent guaranteeing the exponential stability by a common Lyapunov functional (CLF). In fact, we deeply realize that common Lyapunov functional (CLF) may not satisfy all subsystems and become conservative for switched systems. The multi-Lyapunov-Krasovskii functional (MLKF) and delay-dependent method are better choices. They provide a powerful framework for analyzing the stability of switched nonlinear systems with interval time-varying delay.

Remark 5 We now summarize the controller design procedures as follows.

Step 1: The desired convergence rates $\alpha$ and $\beta$ are given. Choose a positive and appropriate parameter $\mu$.

Step 2: Define the variables $X_{i}, S_{1 i}, S_{2 i}, S_{3 i}, T_{1 i}, T_{2 i}, Y_{i}, i \in\{1,2\}$ to be solved.

Step 3: Describe the block form to give a linear matrix inequalities (LMIs).

- $X_{i}>0, S_{1 i}>0, S_{2 i}>0, S_{3 i}>0, T_{1 i}>0, T_{2 i}>0$;

- (36), (37), and (38) are established

Step 4: Complete LMIs model description.

Step 5: Solve LMIs problems.

Step 6: Solve (42).

Then the obtained feedback controller will make the desired performance indices be satisfied.

\section{Numerical example}

In this section, a numerical example is presented to confirm the effectiveness of the proposed approach.

Example 1 Consider system (1) composed of two subsystems with the following parameters:

$$
\begin{aligned}
& A_{11}=\left[\begin{array}{cc}
-0.85 & 0.1 \\
0 & -0.9
\end{array}\right], \quad A_{21}=\left[\begin{array}{cc}
-0.75 & 0 \\
0.1 & -0.8
\end{array}\right], \quad B_{1}=\left[\begin{array}{l}
0.4 \\
0.5
\end{array}\right] \text {, } \\
& A_{12}=\left[\begin{array}{cc}
-0.75 & 0 \\
0.2 & -0.9
\end{array}\right], \quad A_{22}=\left[\begin{array}{cc}
-0.7 & 0.15 \\
0 & -0.85
\end{array}\right], \quad B_{2}=\left[\begin{array}{l}
0.6 \\
0.5
\end{array}\right] \text {, } \\
& D_{1}=\left[\begin{array}{cc}
-0.3 & 0 \\
0.1 & -0.4
\end{array}\right], \quad D_{2}=\left[\begin{array}{cc}
-0.3 & 0 \\
0 & -0.2
\end{array}\right], \quad C_{1}=\left[\begin{array}{cc}
0.3 & 0 \\
0 & 0.4
\end{array}\right] \text {, } \\
& V_{1}=\left[\begin{array}{cc}
-0.65 & 0 \\
0.05 & -0.7
\end{array}\right], \quad V_{2}=\left[\begin{array}{cc}
-0.4 & 0.1 \\
0 & -0.6
\end{array}\right], \quad C_{2}=\left[\begin{array}{cc}
0.4 & 0 \\
0 & 0.3
\end{array}\right] \text {, } \\
& \Lambda_{1}=\left[\begin{array}{cc}
-0.8 & 0.1 \\
0 & -0.7
\end{array}\right], \quad \Lambda_{2}=\left[\begin{array}{cc}
-0.5 & 0.1 \\
0 & -0.4
\end{array}\right] \text {. }
\end{aligned}
$$


Table 1 Calculated upper delay bound $h_{M}$

\begin{tabular}{llllll}
\hline$\alpha$ & 0.55 & 0.6 & 0.65 & 0.7 & 0.75 \\
\hline$h_{M}(\beta=0.7)$ & 0.677 & 0.733 & 0.827 & 0.889 & 0.935 \\
$h_{M}(\beta=0.75)$ & 0.746 & 0.762 & 0.841 & 0.865 & 1.016 \\
$h_{M}(\beta=0.8)$ & 0.804 & 0.852 & 0.906 & 1.119 & 1.352 \\
\hline
\end{tabular}

Let $\alpha=0.45, \beta=0.65, \mu=1.25, h_{m}=0.1, h_{M}=0.6, h=0.5, h(t)=0.5 \sin (t)+0.1, \tau_{d}=0.2$. According to (13), we get the average dwell time

$$
\tau_{a}>\tau_{a}^{*}=\frac{\ln \mu+(\alpha+\beta) \tau_{d}}{\alpha}=0.9848
$$

Choose

$$
\begin{aligned}
& f_{1}(t, x(t), x(t-h(t)))=\left(\begin{array}{c}
0.2 e^{-4 t} \\
0.1 \sin \left(x_{2}(t)\right)
\end{array}\right), \\
& f_{2}(t, x(t), x(t-d(t)))=\left(\begin{array}{c}
0.1 \sin \left(x_{1}(t-h(t))\right) \\
0.1 e^{-3 t}
\end{array}\right) .
\end{aligned}
$$

By solving (36), (37), and (38), we can get

$$
\begin{array}{ll}
X_{1}=\left[\begin{array}{cc}
0.5817 & -0.2809 \\
-0.2809 & 0.5537
\end{array}\right], & X_{2}=\left[\begin{array}{ll}
0.5539 & 0.2561 \\
0.2561 & 0.5298
\end{array}\right], \\
S_{11}=\left[\begin{array}{cc}
1.4886 & -0.3040 \\
-0.3040 & 1.0582
\end{array}\right], & S_{12}=\left[\begin{array}{ll}
1.6720 & 0.0467 \\
0.0467 & 1.3186
\end{array}\right], \\
S_{21}=\left[\begin{array}{cc}
1.7736 & -0.0015 \\
-0.0015 & 1.3371
\end{array}\right], & S_{22}=\left[\begin{array}{ll}
1.4761 & 0.1202 \\
0.1202 & 1.2674
\end{array}\right], \\
S_{31}=\left[\begin{array}{cc}
1.2352 & -0.0103 \\
-0.0103 & 0.9825
\end{array}\right], & S_{32}=\left[\begin{array}{cc}
1.1326 & -0.0831 \\
-0.0831 & 1.1141
\end{array}\right], \\
T_{11}=\left[\begin{array}{cc}
1.2828 & -0.1263 \\
-0.1263 & 1.1729
\end{array}\right], & T_{12}=\left[\begin{array}{ll}
1.2508 & 0.0585 \\
0.0585 & 1.1796
\end{array}\right], \\
T_{21}=\left[\begin{array}{cc}
1.7573 & 0.0240 \\
0.0240 & 1.3964
\end{array}\right], & T_{22}=\left[\begin{array}{cc}
1.5633 & 0.1690 \\
0.1690 & 1.3723
\end{array}\right], \\
Y_{1}=\left[\begin{array}{ll}
0.8214 & 0.4447
\end{array}\right], & Y_{2}=\left[\begin{array}{ll}
0.6154 & 0.7919
\end{array}\right] .
\end{array}
$$

Then the controller gains constructed by (39) are

$$
K_{1}=\left[\begin{array}{ll}
2.3840 & 2.0128
\end{array}\right], \quad K_{2}=\left[\begin{array}{ll}
0.5408 & 1.2334
\end{array}\right] .
$$

By Theorem 2, the maximum value of interval time-varying delay $h_{M}$ for the switched systems (1) is provided in Table 1 for different values of $\alpha$ and $\beta$.

In order to show the effectiveness of the proposed method, the responses of state trajectories of the open-loop and closed-loop systems and switching signals (system signals 
Figure 1 Responses of state trajectories of an open-loop system

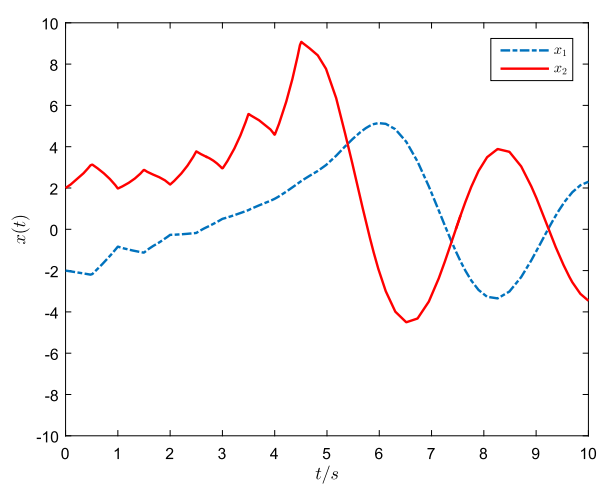

Figure 2 Responses of state trajectories of a closed-loop system

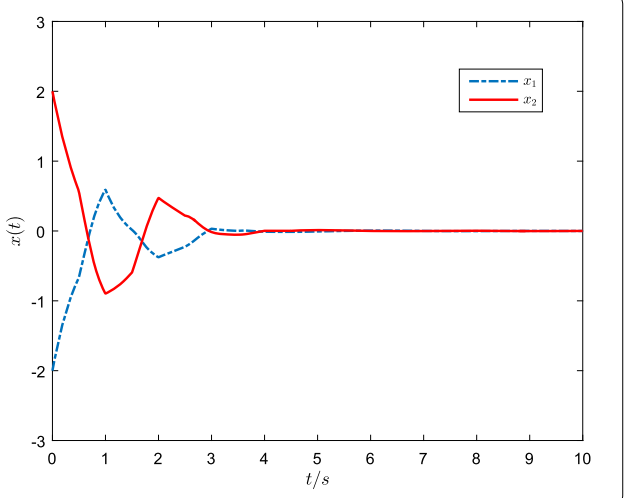

Figure 3 The switching law

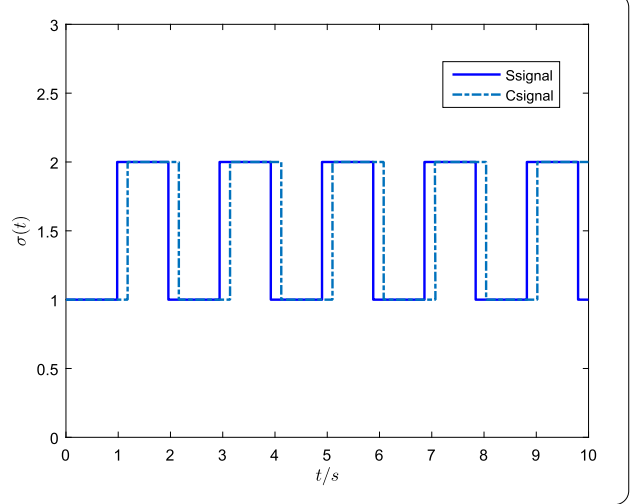

and controller signals) are given in Figs. 1, 2, and 3, respectively. It is clear that the openloop system with initial state $x(0)=(-2,2)^{T}$ is not stable form in Fig. 1. The closed-loop system with initial state $x(0)=(-2,2)^{T}$ is mean-square exponentially stabilizable under the designed asynchronous switching and controllers form in Fig. 2 and 3. Therefore, the effectiveness of the designed asynchronous switching and controllers is fully illustrated.

Example 2 The problem of water pollution is an important issue facing every country, and its development is of great significance to social development. In this section, an example of applying this system to water pollution control systems will be demonstrated.

To facilitate the creation of models for water pollution control systems, we record $p(t)$ and $q(t)$ as the concentrations per unit volume of biochemical oxygen demand and dis- 
solved oxygen, respectively. Simultaneously, let $p^{*}$ and $q^{*}$ indicate the desired steady values of $p(t)$ and $q(t)$ in a reach of a polluted river, respectively. Moreover, we take $p^{*}$ and $q^{*}$ as corresponding to some measure of water quality standards, given by the following definition:

$$
x_{1}(t)=p(t)-p^{*}, \quad x_{2}(t)=q(t)-q^{*}, \quad x(t)=\left[x_{1}^{T}(t) x_{2}^{T}(t)\right]^{T} .
$$

As a result, the dynamic equation for $x(t)$ can be expressed as

$$
d x(t)=[A x(t)+\bar{A} x(t-h(t))+B u(t)] d t+x(t) d \omega(t)
$$

where

$$
A=\left[\begin{array}{cc}
-m_{1}-\varepsilon_{1}-\varepsilon_{2} & 0 \\
-m_{3} & -m_{2}-\varepsilon_{1}-\varepsilon_{2}
\end{array}\right], \quad \bar{A}=\left[\begin{array}{cc}
\varepsilon_{2} & 0 \\
0 & \varepsilon_{2}
\end{array}\right], \quad B=\left[\begin{array}{c}
\varepsilon_{1} \\
1
\end{array}\right],
$$

$m_{i}(i=1,2,3), \varepsilon_{1}$ and $\varepsilon_{2}$ are known constants, and $\omega(t)$ is a one-dimensional Brownian motion that satisfies condition (3). Moreover, $u(t)=\left[u_{1}^{T}(t) u_{2}^{T}(t)\right]^{T}$ is the control variable of river pollution system. We can learn the engineering significance of these parameters from [1]. This paper assumes that system actuators have good performance or failure, and according to the actual situation, we know that at least one actuator can ensure the normal operation of the river pollution system. In addition, for simulation of our purposes, we do not consider the nonlinear perturbation term, and the nonlinear perturbation term is not also considered in [26]. As a consequence, the river pollution system (43) can be modeled as a switched system consisting of two subsystems:

$$
\begin{array}{ll}
d x(t)=\left[A_{11} x(t)+A_{21} x(t-h(t))+B_{1} u(t)\right] d t+x(t) d \omega(t), & \text { no failures occur } \\
d x(t)=\left[A_{12} x(t)+A_{22} x(t-h(t))+B_{2} u(t)\right] d t+x(t) d \omega(t), & \text { failures occur. }
\end{array}
$$

Next, we choose $m_{1}=1.1, m_{2}=0.6, m_{3}=1.3, \varepsilon_{1}=0.5, \varepsilon_{2}=0.4$ and get that

$$
A=\left[\begin{array}{cc}
-2 & 0 \\
-1.3 & -1.5
\end{array}\right], \quad \bar{A}=\left[\begin{array}{cc}
0.4 & 0 \\
0 & 0.4
\end{array}\right], \quad B=\left[\begin{array}{c}
0.5 \\
1
\end{array}\right] .
$$

Let $\alpha=0.5, \beta=0.6, \mu=1.15, h_{m}=0.15, h_{M}=0.65, h=0.3, h(t)=0.3 \sin (t)+0.2, \tau_{d}=0.1$.

By (36), (37), and (38), we have

$$
\begin{array}{ll}
X_{1}=\left[\begin{array}{ll}
0.3725 & 0.1019 \\
0.1019 & 0.6832
\end{array}\right], & X_{2}=\left[\begin{array}{ll}
0.4827 & 0.1024 \\
0.1024 & 0.6728
\end{array}\right], \\
S_{11}=\left[\begin{array}{ll}
1.2871 & 0.2028 \\
0.2028 & 0.9811
\end{array}\right], & S_{12}=\left[\begin{array}{ll}
1.1728 & 0.1422 \\
0.1422 & 1.1107
\end{array}\right], \\
S_{21}=\left[\begin{array}{ll}
1.2821 & 0.0102 \\
0.0102 & 1.1371
\end{array}\right], & S_{22}=\left[\begin{array}{ll}
1.0963 & 0.3294 \\
0.3294 & 1.1623
\end{array}\right], \\
S_{31}=\left[\begin{array}{ll}
1.4372 & 0.2031 \\
0.2031 & 1.0921
\end{array}\right], & S_{32}=\left[\begin{array}{ll}
1.2355 & 0.2832 \\
0.2832 & 1.2362
\end{array}\right],
\end{array}
$$


Figure 4 State response of subsystem 1

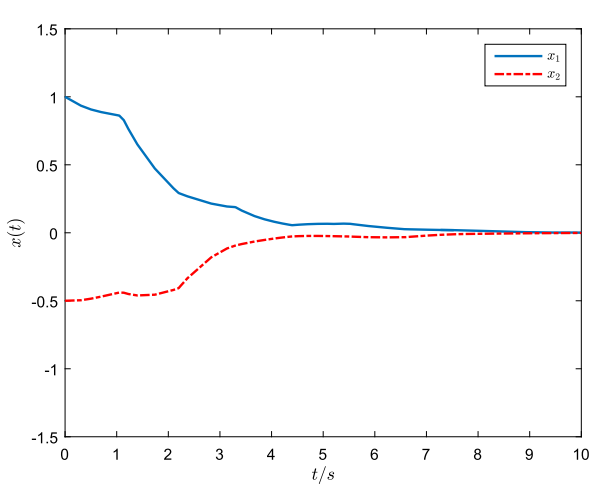

Figure 5 State response of subsystem 2

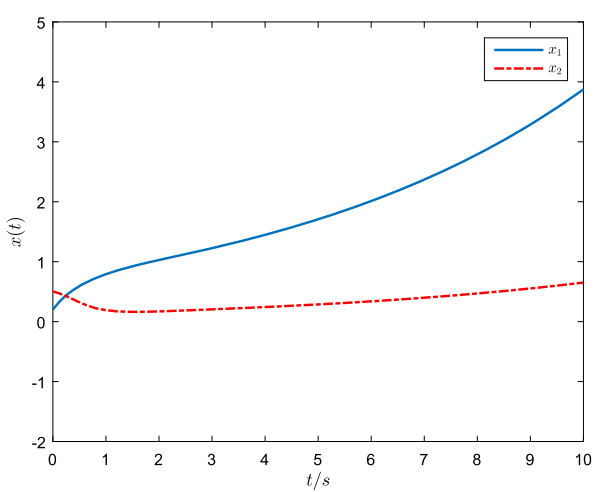

$$
\begin{array}{ll}
T_{11}=\left[\begin{array}{ll}
1.0812 & 0.2733 \\
0.2733 & 1.0128
\end{array}\right], & T_{12}=\left[\begin{array}{ll}
1.3562 & 0.0738 \\
0.0738 & 1.3792
\end{array}\right], \\
T_{21}=\left[\begin{array}{ll}
1.3783 & 0.0367 \\
0.0367 & 1.0917
\end{array}\right], & T_{22}=\left[\begin{array}{ll}
1.4923 & 0.2623 \\
0.2623 & 1.8392
\end{array}\right], \\
Y_{1}=\left[\begin{array}{ll}
0.9972 & 0.5377
\end{array}\right], & Y_{2}=\left[\begin{array}{ll}
0.8362 & 0.5849
\end{array}\right] .
\end{array}
$$

Then the controller gains constructed by (39) are

$$
K_{1}=\left[\begin{array}{ll}
2.5665 & 0.4042
\end{array}\right], \quad K_{2}=\left[\begin{array}{ll}
1.5996 & 0.6259
\end{array}\right] .
$$

Figure 4 describes state response of subsystem 1 with the initial condition $x(0)=(1,-0.5)^{T}$. Figure 5 describes state response of subsystem 2 with the initial condition $x(0)=(0.2,0.5)^{T}$. Through the designed switching signal and our approach, we can get that system (43) with the initial condition $x(0)=(2,-2)^{T}$ is mean-square exponentially stabilizable for any switching signal under the feedback control form Fig. 6. As a consequence, this verifies the effectiveness of our results in the control of river pollution process.

\section{Conclusions}

The switching signal of the switched controller involves delay, which results in the asynchronous switching between the candidate controllers and subsystems. In the paper, we 
Figure 6 State response of system (43)

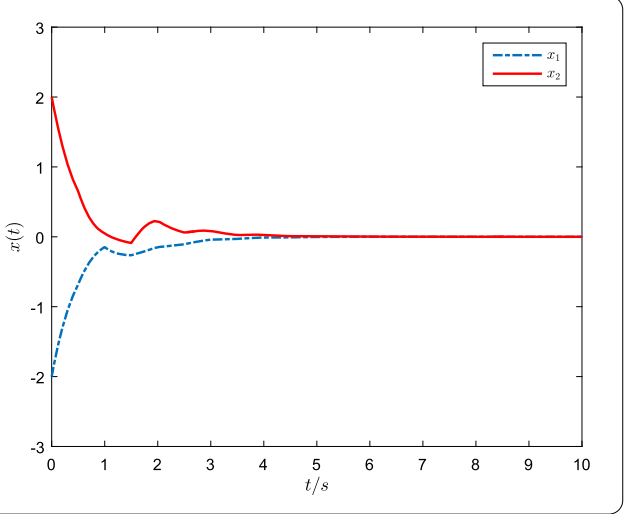

have investigated the problem of asynchronous switching for nonlinear switched stochastic systems with interval time-varying delay based on time-dependent switching signal for the matched and mismatched sections, respectively. By constructing a new multiLyapunov-Krasovskii functional, which is related to the size of the time delay, using the matrix inequality technique and the average dwell time approach, the mean-square exponential stabilization criteria for nonlinear switched stochastic systems with interval timevarying delay are obtained under asynchronous switching. Then, the proposed approach is extended to design state feedback controller for switched stochastic systems by special operations of matrices. Finally, the numerical example illustrates the effectiveness of the theoretical results. Compared with the existing results, the new condition is less conservative. In order to better study the asynchronous switching issue, our future work will focus on extending the proposed method to a delay-dependent robust dissipative problem for a class of nonlinear switched systems with mixed delays and the stabilization of stochastic switched nonlinear systems with Markov jumps.

Acknowledgements

The author would like to thank the editor and the anonymous reviewers for their valuable comments and suggestions to improve this paper.

Funding

This work was supported by the National Natural Science Foundation for Young Scientists of China (Grant No. 71502050), the Key Scientific Research Projects of Colleges and Universities of Henan Province (No. 18A630001, 18A110008, and 18A110010)

Availability of data and materials

Data sharing not applicable to this article as no datasets were generated or analysed during the current paper.

Ethics approval and consent to participate

Not applicable.

Competing interests

The author declares that there are no competing interests.

Consent for publication

Not applicable.

Authors' contributions

The author read and approved the final version of the manuscript.

\section{Publisher's Note}

Springer Nature remains neutral with regard to jurisdictional claims in published maps and institutional affiliations. 


\section{References}

1. Yu, P.A., et al.: Introduction to Control of Oscillations and Chaos. World Scientific, Singapore (1998)

2. Li, H.: Sampled-data state estimation for complex dynamical networks with time-varying delay and stochastic sampling. Neurocomputing 138, 78-85 (2014)

3. Bishop, B.E., Spong, M.W.: Control of redundant manipulators using logic-based switching. In: Proceedings of the 37th IEEE Conference on Decision and Control, vol. 2, pp. 1488-1493. IEEE Press, New York (1998)

4. Liberzon, D.: Switching in Systems and Control. Birkhäuser, Boston (2003)

5. Zhang, L., Zhu, Y., Ning, Z., Yin, X.: Resilient estimation for networked systems with variable communication capability. IEEE Trans. Autom. Control 61(12), 4150-4156 (2016)

6. Zhang, J., Ma, L., Liu, Y.: Passivity analysis for discrete-time neural networks with mixed time-delays and randomly occurring quantization effects. Neurocomputing 216, 657-665 (2016)

7. Ma, L., Wang, Z., Han, Q.-L., Liu, Y.: Dissipative control for nonlinear Markovian jump systems with actuator failures and mixed time-delays. Automatica 98, 358-362 (2018)

8. Kim, D.K., Park, P., Ko, J.W.: Output-feedback $h_{\infty}$ control of systems over communication networks using a deterministic switching system approach. Automatica 40(7), 1205-1212 (2004)

9. Jeon, D., Tomizuka, M.: Learning hybrid force and position control of robot manipulators. IEEE Trans. Robot. Autom. 9(4), 423-431 (1993)

10. Zhang, L., Shi, P.: Stability, $l_{2}$-gain and asynchronous $h_{\infty}$ control of discrete-time switched systems with average dwell time. IEEE Trans. Autom. Control 54(9), 2192-2199 (2009)

11. Zhu, Y., Zhong, Z., Basin, M.V., Zhou, D.: A descriptor system approach to stability and stabilization of discrete-time switched PWA systems. IEEE Trans. Autom. Control 63(10), 3456-3463 (2018)

12. Xiang, W., Tran, H.-D., Johnson, T.T.: Robust exponential stability and disturbance attenuation for discrete-time switched systems under arbitrary switching. IEEE Trans. Autom. Control 63(5), 1450-1456 (2018)

13. Su, X., Shi, P., Wu, L., Song, Y., et al.. Fault detection filtering for nonlinear switched stochastic systems. IEEE Trans. Autom. Control 61(5), 1310-1315 (2016)

14. Sun, T., Zhou, D., Zhu, Y., Basin, M.V.: Stability, L2-gain analysis, and parity space-based fault detection for discrete-time switched systems under dwell-time switching. In: IEEE Transactions on Systems, Man, and Cybernetics: Systems (2018)

15. Ma, L., Wang, Z., Liu, Y., Alsaadi, F.E.: A note on guaranteed cost control for nonlinear stochastic systems with input saturation and mixed time-delays. Int. J. Robust Nonlinear Control 27(18), 4443-4456 (2017)

16. Wang, H., Liu, P.X., Niu, B.: Robust fuzzy adaptive tracking control for nonaffine stochastic nonlinear switching systems. IEEE Trans. Cybern. 48(8), 2462-2471 (2018)

17. Duan, C., Wu, F: Analysis and control of switched linear systems via dwell-time min-switching. Syst. Control Lett. 70 , 8-16 (2014)

18. Chen, H., Shi, P., Lim, C.-C.: Stability of neutral stochastic switched time delay systems: an average dwell time approach. Int. J. Robust Nonlinear Control 27(3), 512-532 (2017)

19. Xie, L.: Output feedback $h_{\infty}$ control of systems with parameter uncertainty. Int. J. Control 63(4), 741-750 (1996)

20. Tian, Y., Cai, Y., Sun, Y: Stability of switched nonlinear time-delay systems with stable and unstable subsystems. Nonlinear Anal. Hybrid Syst. 24, 58-68 (2017)

21. Dong, Y., Wang, X., Mei, S., Li, W.: Exponential stabilization of nonlinear uncertain systems with time-varying delay J. Eng. Math. 77(1), 225-237 (2012)

22. Dong, Y., Li, T., Mei, S.: Exponential stabilization and L2-gain for uncertain switched nonlinear systems with interval time-varying delay. Math. Methods Appl. Sci. 39(13), 3836-3854 (2016)

23. Lien, C.-H.: Delay-dependent and delay-independent guaranteed cost control for uncertain neutral systems with time-varying delays via LMI approach. Chaos Solitons Fractals 33(3), 1017-1027 (2007)

24. Sun, X.-M., Zhao, J., Hill, D.J.: Stability and L2-gain analysis for switched delay systems: a delay-dependent method. Automatica 42(10), 1769-1774 (2006)

25. Wang, X., She, K., Zhong, S., Yang, H.: New result on synchronization of complex dynamical networks with time-varying coupling delay and sampled-data control. Neurocomputing 214, 508-515 (2016)

26. Wang, Y.-E., Wu, B.-W., Wu, C.: Stability and L2-gain analysis of switched input delay systems with unstable modes under asynchronous switching. J. Franklin Inst. 354(11), 4481-4497 (2017)

27. Wang, X., Zong, G., Sun, H.: Asynchronous finite-time dynamic output feedback control for switched time-delay systems with non-linear disturbances. IET Control Theory Appl. 10(10), 1142-1150 (2016)

28. Lian, J., Ge, Y., Han, M.: Stabilization for switched stochastic neutral systems under asynchronous switching. Inf. Sci. 222, 501-508 (2013)

29. Wang, Y.-E., Zhao, J., Jiang, B.: Stabilization of a class of switched linear neutral systems under asynchronous switching. IEEE Trans. Autom. Control 58(8), 2114-2119 (2013)

30. Lian, J., Ge, Y.: Robust $h_{\infty}$ output tracking control for switched systems under asynchronous switching. Nonlinear Anal. Hybrid Syst. 8, 57-68 (2013)

31. Wang, R., Shi, P., Wu, Z.-G., Sun, Y.-T.: Stabilization of switched delay systems with polytopic uncertainties under asynchronous switching. J. Franklin Inst. 350(8), 2028-2043 (2013)

32. Wang, Y.: Exponential stabilization for a class of nonlinear switched systems with mixed delays under asynchronous switching. Complexity 2018, 15 (2018)

33. Zhao, X., Zhang, L., Shi, P., Liu, M.: Stability of switched positive linear systems with average dwell time switching. Automatica 48(6), 1132-1137 (2012)

34. Li, S., Xiang, Z: Stabilisation of a class of positive switched nonlinear systems under asynchronous switching. Int. J. Syst. Sci. 48(7), 1537-1547 (2017)

35. Xiang, Z., Wang, R:: Robust control for uncertain switched non-linear systems with time delay under asynchronous switching. IET Control Theory Appl. 3(8), 1041-1050 (2009)

36. Xiang, Z., Wang, R., Chen, Q.: Robust reliable stabilization of stochastic switched nonlinear systems under asynchronous switching. Appl. Math. Comput. 217(19), 7725-7736 (2011) 